\title{
Effect of DEM sources on quality indicators of predictive maps of soil cover
}

\author{
V. R. Cherlinka ${ }^{\star \star}$, Y. M. Dmytruk¹, Y. H. Bodyan² \\ ${ }^{1}$ Yuriy Fedkovych Chernivtsi National University, Chernivtsi, Ukraine \\ ${ }^{2}$ Chernivtsi branch of the State Institution "Soils Protection Institute of Ukraine", Chernivtsi, Ukraine
}

\begin{tabular}{|c|c|}
\hline ARTICLE INFO & ABSTRACT \\
\hline $\begin{array}{l}\text { Received } 01.07 .2020 \\
\text { Received in revised } \\
\text { form } 08.08 .2020 \\
\text { Accepted } 15.09 .2020 \\
\text { Available online } \\
05.11 .2020 \\
\end{array}$ & $\begin{array}{l}\text { The aim of the study was to identify the impact of digital elevation models of different origins } \\
\text { on the qualitative characteristics of forecast maps of soil cover or cartograms of agro- } \\
\text { production soil groups using predictive modeling technologies. The current situation with } \\
\text { large-scale soil cartographic data in Ukraine is analyzed and it is shown that the fastest and } \\
\text { most cost-effective way to fill gaps in creating a continuous cartographic coverage for }\end{array}$ \\
\hline $\begin{array}{l}\text { Keywords: } \\
\text { cartogram of agro- } \\
\text { production soil groups; } \\
\text { DEM; } \\
\text { digital elevation model; } \\
\text { modelling; } \\
\text { morphometric } \\
\text { parameters, } \\
\text { predicative algorithms; } \\
\text { soil map }\end{array}$ & $\begin{array}{l}\text { based on morphometric analysis of digital elevation models, which distinguishes a number } \\
\text { of predictors, which are further analyzed for links with existing cartographic soil materials by } \\
\text { creating a mathematical predictive model using landscape reference points and associated } \\
\text { soil type. The identified difference in the quality of predictive materials using the Cohen's } \\
\text { kappa coefficient allows us to recommend individual sources of DEM as a basis for such } \\
\text { tasks. A demonstration of a closed production cycle of creating predicative soil cartographic } \\
\text { materials based on free software (GRASS and Quantum geographic information systems, } \\
\text { language and environment for statistical computing and graphics R and shareware - Easy } \\
\text { Trace vectorizer) was conducted. }\end{array}$ \\
\hline
\end{tabular}

${ }^{*}$ Corresponding author: v.cherlinka@chnu.edu.ua

Citing: Cherlinka V.R., Dmytruk Y.M., Bodyan Y.H. 2020. Effect of DEM sources on quality indicators of predictive maps of soil cover. Agrochemistry and Soil Science. Collected papers. No. 90. Kharkiv: NSC ISSAR, P. 36-46. DOI: https://doi.org/10.31073/acss90-04.

\section{References}

1. Achasov A.B., Titenko G.V., Kurilov V.I. 2015. Data of remote sensing as a basis of soil mapping: economic aspect. Bulletin of VN Karazin Kharkiv National University. Series: Ecology (1104. Issue 10), 60-66. (Ukr.). URL http://journals.uran.ua/visnukkhnu ecology/article/download/25458/33191.

2. Cherlinka V. 2017. Using Geostatistics, DEM and Remote Sensing to Clarify Soil Cover Maps of Ukraine. In: Dent D., Dmytruk Y. (Eds.), Soil Science Working for a Living: Applications of soil science to present-day problems. Springer-Verlag GmbH, Cham, Switzerland, Ch. 7. P. 89100. URL: https://ink.springer.com/chapter/10.1007/978-3-319-45417-7 7.

3. Cherlinka V., Dmytruk Y., Barabas D. 2019. Methods of verification of soils prediction maps: a case study from Chernivtsi region, Ukraine. Geographia Cassoviensis. Vol. 13, no. 2. P. 141-160. DOI: https://doi.org/10.33542/GC2019-2-04.

4. Cherlinka V.R., Lobova O.V. 2018. Methodical approaches to the coordination of soil cartographic materials on the borders of administrative-territorial units of Ukraine. Scientific reports of NULES of Ukraine. №6(76). P. 1-15. DOI: https://doi.org/10.31548/dopovidi2018.06.010 (Ukr.).

5. Polchyna S.M., Nikorych V.A., Danchu O.A. 2004. Application of the modern FAO / WRB soil classification system to the soil cover map of Chernivtsi region. Soil Science. Vol. 5(1-2). P. 27-33. URL: http://arr.chnu.edu.ua/jspui/bitstream/123456789/471/1/Nikorich.pdf.

6. Browning D.M., Duniway M.C. 2011. Digital soil mapping in the absence of field training data: A case study using terrain attributes and semiautomated soil signature derivation to distinguish ecological potential. Applied and Environmental Soil Science. DOI: https://doi.org/10.1155/2011/421904.

7. Brungard C.W., Boettinger J.L., Duniway M.C., Wills S.A., Edwards T.C. 2015. Machine learning for predicting soil classes in three semiarid landscapes. Geoderma. Vol. 239. P. 68-83. DOI: https://doi.org/10.1016/j.geoderma.2014.09.019.

8. Caten A.T., Dalmolin R.S.D., Pedron F.D.A., Ruiz L.F.C., Silva C.A.D. 2013. An appropriate data set size for digital soil mapping in Erechim, Rio Grande do Sul, Brazil. Revista Brasileira de Ciência do Solo. 37(2). P. 359-366. DOI: https://doi.org/10.1590/s010006832013000200007

9. Heung B., Ho H.C., Zhang J., Knudby A., Bulmer C.E., Schmidt M.G. 2016. An overview and comparison of machine-learning techniques for classification purposes in digital soil mapping. Geoderma. Vol. 265. P. 62-77. DOI: https://doi.org/10.1016/j.geoderma.2015.11.014.

10. Heung B., Hodúl M., Schmidt M.G. 2017. Comparing the use of training data derived from legacy soil pits and soil survey polygons for mapping soil classes. Geoderma. Vol. 290. P. 51-68. DOI: https://doi.org/10.1016/j.geoderma.2016.12.001.

11. MacMillan R. 2008. Experiences with Applied DSM: Protocol, Availability, Quality and Capacity Building. In: Hartemink A.E., McBratney A., Mendonça-Santos M. (eds) Digital Soil Mapping with Limited Data. Springer, Dordrecht. P. 113-135. DOI: https://doi.org/10.1007/978-1-4020-8592-5_10.

12. Malone B.P., Minasny B., McBratney A.B. 2016. Using R for Digital Soil Mapping. Progress in Soil Science. Springer International Publishing. DOI: https://doi.org/10.1007/978-3-319-44327-0.

13. McBratney A.B., Santos M.L.M., Minasny B. 2003. On digital soil mapping. Geoderma. Vol. 117(1-2). P. 3-52. DOI: https://doi.org/10.1016/s0016-7061(03)00223-4.

14. Scull P., Franklin J., Chadwick O. A., McArthur D. 2003. Predictive soil mapping: a review. Progress in Physical Geography. 27(2). P. 171-197. DOI: https://doi.org/10.1191/0309133303pp366ra.

15. Walter C., Lagacherie P., Follain S. 2006. Integrating pedological knowledge into digital soil mapping. In: Lagacherie P., McBratney A.B., Voltz M. (Eds.). Digital Soil Mapping: An Introductory Perspective. Vol. 31 of Developments in Soil Science. Elsevier, Amsterdam. Ch. 22. P. 281301. DOI: https://doi.org/10.1016/s0166-2481(06)31022-7.

16. Florinsky I.V. 2016. Digital Terrain Analysis in Soil Science and Geology. 2 edition. Amsterdam : ACADEMIC PRESS / Elsevier. 506 p. https://doi.org/10.1016/c2015-0-02363-2.

17. Lagacherie P., Robbez-Masson J.M., Nguyen-The N., Barthès J.P. 2001. Mapping of reference area representativity using a mathematical soils cape distance. Geoderma. Vol. 101(3-4). P. 105-118. DOI: https://doi.org/10.1016/S0016-7061(00)00101-4. 
18. Giasson E., Figueiredo S.R., Tornquist C.G., Clarke R.T. 2008. Digital soil mapping using logistic regression on terrain parameters for several ecological regions in Southern Brazil. In: Hartemink A.E., McBratney A.B., de Lourdes Mendonça-Santos M. (Eds.), Digital Soil Mapping with Limited Data. Springer Netherlands, Amsterdam, Ch. 19. P. 225-232. DOI: https://doi.org/10.1007/978-1-4020-8592-5_19.

19. Kempen B., Brus D.J., Heuvelink G.B.M., Stoorvogel J.J. 2009. Updating the 1:50,000 Dutch soil map using legacy soil data: A multinomial logistic regression approach. Geoderma. Vol. 151(3). P. 311-326. DOI: https://doi.org/10.1016/j.geoderma.2009.04.023.

20. Debella-Gilo M., Etzelmüller B., 2009. Spatial prediction of soil classes using digital terrain analysis and multinomial logistic regression modeling integrated in GIS: Examples from Vestfold County, Norway. Catena. Vol. 77(1). P. 8-18. DOI: https://doi.org/10.1016/j.catena.2008.12.001.

21. Hengl, T., 2009. A practical guide to geostatistical mapping, 2nd Edition. Office for Official Publications of the European Communities, Luxembourg. URL http://spatial-analyst.net/book/system/files/Hengl_2009_GEOSTATe2c1w.pdf.

22. Cherlinka V.R. 2017. Variations of predictive efficiency of soil maps depending on the methods of constructing educational samples of predicative algorithms. Ecology and noospherology. T. 28(3-4). P. 55-71. (Ukr.) http://erae.dp.ua/index.php/erae/article/view/20.

23. Esfandiarpour-Boroujeni I., Shahini-Shamsabadi M., Shirani H., Mosleh Z., Bagheri-Bodaghabadi M., Salehi M.H. 2020. Assessment of different digital soil mapping methods for prediction of soil classes in the Shahrekord plain, Central Iran. Catena, Vol. 193. Art.104648. DOI: https://doi.org/10.1016/j.catena.2020.104648.

24. Kidd D., Searle R., Grundy M., McBratney A., Robinson N., O'Brien L., Jones E. 2020. Operationalising digital soil mapping - Lessons from Australia. Geoderma Regional. DOI: https://doi.org/10.1016/j.geodrs.2020.e00335.

25. Batjes N.H., Ribeiro E., van Oostrum A. 2020. Standardised soil profile data to support global mapping and modelling (WoSIS snapshot 2019). Earth Syst. Sci. Data. Vol. 12. P. 299-320. DOI: https://doi.org/10.5194/essd-12-299-2020.

26. Hengl T., Mendes de Jesus J., Heuvelink G.B.M., Ruiperez Gonzalez M., Kilibarda M., Blagotić A. [et al.]. 2017. SoilGrids250m: Global gridded soil information based on machine learning. PLOS ONE. Vol. 12(2): DOI: https://doi.org/10.1371/journal.pone.0169748.

27. Chen Z., Ye F., Fu W., Ke Y., Hong H. 2020. The influence of DEM spatial resolution on landslide susceptibility mapping in the Baxie River basin, NW China. Natural Hazards, 101. P.853-877. DOI: https://doi.org/10.1007/s11069-020-03899-9.

28. Munoth P., Goyal R. 2019. Effects of DEM source, spatial resolution and drainage area threshold values on hydrological modeling. Water Resources Management. Vol. 33(9). P. 3303-3319. DOI: https://doi.org/10.1007/s11269-019-02303-x.

29. Cherlinka V.R. 2017. Influence of resolution of digital relief models on the quality of predicative simulation of soil cover. Soil Science. T. 18. №1-2. P. 79-95. URL: http://www.dnu.dp.ua/docs/zbirniki/fbem/program_5cf026b4bbf65.pdf. (Ukr.).

30. Danielson J.J., Gesch D.B. 2011. Global multi-resolution terrain elevation data 2010 (GMTED2010) (p. 26). US Department of the Interior, US Geological Survey. DOI: https://doi.org/10.5066/F7J38R2N.

31. NASA/METI/AIST/Japan Spacesystems, and U.S./Japan ASTER Science Team. ASTER Global Digital Elevation Model V003. 2019, distributed by NASA EOSDIS Land Processes DAAC. DOI: https://doi.org/10.5067/ASTER/ASTGTM.003. Accessed 2020-09-29.

32. NASA JPL. NASA Shuttle Radar Topography Mission Global 1 arc second. 2013, distributed by NASA EOSDIS Land Processes DAAC. DOI: https://doi.org/10.5067/MEaSUREs/SRTM/SRTMGL1.003. Accessed 2020-09-09.

33. Takaku J., Tadono T., Tsutsui K., Ichikawa M. 2018. Quality Improvements of 'AW3D'Global Dsm Derived from Alos Prism. In IGARSS 2018-2018 IEEE International Geoscience and Remote Sensing Symposium. P. $1612-1615 . \quad$ IEEE. URL: https://ieeexplore.ieee.org/document/8518360.

34. EU-DEM Upgrade Documentation EEA User Manual. Available online: https://land.copernicus.eu/user-corner/technical-library/eu-dem-v11-user-guide (accessed on 5 September 2020).

35. Cherlinka V.R. Digital elevation models in soil science: theoretical and methodological bases and practical application: Extended Abstract of Dr. Biol. Sciences: 03.00. Dissertation, Chernivtsi, Yuriy Fedkovych Chernivtsi National University, 2019.538 p. (Ukr.). https://drive.google.com/open?id=1TZubbaD3fNIk7FQUkSyZOdK_dPpozqpN.

36. Kameneva T. 2020. Formation of the National Infrastructure of Geospatial Data in Ukraine and its legal regulation. Public opinion on lawmaking. № 9 (194). P. 21-25. (Ukr.) URL: http://nbuviap.gov.ua/images/dumka/2020/9.pdf.

37. Cherlinka V.R. Adaptation of large-scale soil maps to their practical use in GIS. 2015. Agrochemistry and Soil Science. Collected papers. No. 84. ISSAR. Kharkiv. P. 20-28. (Ukr.). URL: http://agrochemsoilsci.org/84/84-03.html.

38. Resolution of the Presidium of the National Academy of Agrarian Sciences of Ukraine No. 09/05 of 26.06.2019 (Minutes №9) on the establishment of the Ukrainian Soil Information Center on the basis of the National Scientific Center "Institute for Soil Science and Agrochemistry Research named after O.N. Sokolovsky" (in Ukrainian). URL: http://www.issar.com.ua/downloads/postanova-1.pdf.

39. Laktionova T.M. 2018. The experience of creating and using seven soil databases in the Soil-Geoeocophysics Laboratory. Agrochemistry and Soil Science. Collected papers. No. 87. ISSAR. Kharkiv. P. 63-71. (Ukr.). DOI: https://doi.org/10.31073/acss87-10

40. Laktionova T.N., Bigun O.N., Nakisko S.G., Uvarenko K.Yu. 2020. Design and functional features of the world's leading soil databases Analytical review. Agrochemistry and Soil Science. Collected papers. No. 89. Kharkiv: NSC ISSAR, P. 4-17. (Ukr.). DOI: https://doi.org/10.31073/acss89-01.

41. GRASS Development Team. 2020. Geographic Resources Analysis Support System (GRASS GIS) Software. Version 7.6. URL: http://grass.osgeo.org.

42. QGIS Development Team, 2020. QGIS Geographic Information System. URL http://qgis.osgeo.org

43. EasyTrace group, 2015. Easy Trace 7.99. Digitizing software. URL http://www.easytrace.com.

44. R Development Core Team. 2020. R: A language and environment for statistical computing. R Foundation for Statistical Computing. URL: http://www.r-project.org.

45. Breiman L. 2001. Random forests. Machine learning 45(1), 5-32. DOI: https://doi.org/10.1023/A:1010933404324.

46. Cutler A., Cutler D.R., Stevens J.R. 2012. Random Forests. Springer US, Boston, MA, P. 157-175. DOI: https://doi.org/10.1007/978-14419-9326-7 5

47. Xu R., Nettleton D., Nordman D.J. 2016. Case-specific random forests. Journal of Computational and Graphical Statistics. 25(1). P. 49-65. DOI: https://doi.org/10.1080/10618600.2014.983641.

48. Cherlinka V.R., Dmytruk Y.M., Zaharovskyy V.S. 2017. Comparative estimation of the accuracy of simulation modeling of soil cover and forecast of cartograms of agro-industrial groups. Biological systems. Vol. 9. Issue. 2. P. 298-306. http://nbuv.gov.ua/UJRN/biolsist_2017_9_2_23.

49. Cherlinka V.R, Dmytruk Y.M. 2014. Problems of creation, georectification and use of large-scale digital elevation models. Geopolitics and

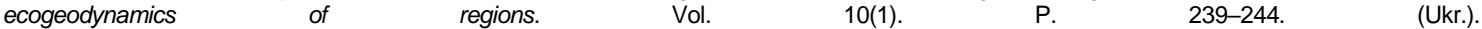
https://www.researchgate.net/publication/296596582_Problemi_stvorenna_georektifikacii_ta_vikoristanna_krupnomasstabnih_cifrovih_modelej_re lefu.

50. Hutchinson M.F. 1995. Interpolating mean rainfall using thin plate smoothing splines. International journal of geographical information systems. Vol. 9(4). P. 385-403. DOI: https://doi.org/10.1080/02693799508902045.

51. Wahba G. 1990. Spline models for observational data. Society for industrial and applied mathematics. DOI: https://doi.org/10.1137/1.9781611970128.

52. USGS Earth Explorer. URL: https://earthexplorer.usgs.gov/.

53. ALOS Global Digital Surface Model "ALOS World 3D - 30m (AW3D30)" URL: https://www.eorc.jaxa.jp/ALOS/en/aw3d30/

54. Stevenson J.A, Sun X., Mitchell N.C. 2009. Despeckling SRTM and other topographic data with a denoising algorithm. Geomorphology. 144. P. 238-252. DOI: https://doi.org/10.1016/j.geomorph.2009.07.006.

55. Sun X., Rosin P.L., Martin R.R., Langbein F.C. 2007. Fast and Effective Feature-Preserving Mesh Denoising. IEEE. Transactions on Visualisation and Computer Graphics. 13(5). P. 925-938. DOI: https://doi.org/10.1109/TVCG.2007.1065. 
56. Wright M.N., Ziegler A. 2017. ranger: A Fast Implementation of Random Forests for High Dimensional Data in C++ and R. Journal of Statistical Software. 77(1). P. 1-17. DOI: https://doi.org/10.18637/jss.v077.i01.

57. Grinand C., Arrouays D., Laroche B., Martin M.P. 2008. Extrapolating regional soil landscapes from an existing soil map: Sampling intensity, validation procedures, and integration of spatial context. Geoderma. Vol. 143(1). P. 180-190. DOI: https://doi.org/10.1016/j.geoderma.2007.11.004

58. Kuhn M. 2008. Building Predictive Models in R Using the caret Package. Journal of Statistical Software. Vol. 28(5). P. 1-26. DOI: https://doi.org/10.18637/jss.v028.105.

59. Landis. J.R., Koch G.G. 1977. The measurement of observer agreement for categorical data. Biometrics. Vol. 33, No. 1. P. 159-174. URL https://doi.org/10.2307/2529310

60. Li W., Zhang C. 2007. A Random-Path Markov Chain Algorithm for Simulating Categorical Soil Variables from Random Point Samples. Soil Science Society of America Journal. Vol. 71(3). P. 656-668. DOI: https://doi.org/10.2136/sssaj2006.0173.

61. Public cadastral map of Ukraine. URL: https://map.land.gov.ua/ (accessed on 5 September 2020) (Ukr.).

62. National (all-Ukrainian) normative monetary valuation of agricultural lands https://ngo.land.gov.ua/uk/map/ (accessed on 5 September 2020) (Ukr.)

63. Cherlinka V.R., Dmytruk Y.M. 2018. Solving existing problems with soil maps in Ukraine. Biological systems. Vol. 10(1) P. 298-308. DOI: https://doi.org/10.31861/biosystems2018.01.094.

УДК 631.4:551.4:004.942

\title{
Вплив джерел ЦМР на якісні показники предикативних карт грунтового покриву
}

\section{В. Р. Черлінка ${ }^{1 *}$, Ю. М. Дмитрук'}

1Чернівецький національний університет ім. Ю. Федьковича, Чернівці, Україна

2Чернівецька філія ДУ «Інститут охорони ґрунтів України», Чернівці, Україна

\begin{tabular}{|c|c|}
\hline ІНФОРМАЦІЯ & АНОТАЦІЯ \\
\hline $\begin{array}{l}\text { Ключові слова: } \\
\text { карта ґрунтів; } \\
\text { картограма } \\
\text { агровиробничих груп } \\
\text { ґрунтів; моделювання; } \\
\text { морфометричні } \\
\text { параметри; } \\
\text { предикативні } \\
\text { алгоритми; цифрова } \\
\text { модель рельєфу; ЦМР }\end{array}$ & $\begin{array}{l}\text { Метою дослідження було виявлення впливу цифрових моделей рельєфу (ЦМР) різного } \\
\text { походження на якісні характеристики прогнозних карт ґрунтового покриву чи картограм } \\
\text { агровиробничих груп ґрунтів за використання технологій предикативного моделювання. } \\
\text { Проаналізовано поточну ситуацію з великомасштабними ґрунтовими картографічними } \\
\text { даними в Україні та показано, що найбільш швидким та економічно ефективним } \\
\text { способом заповнення прогалин при створенні суцільного картографічного покриття для } \\
\text { необстежених територій, площа яких становить до } 33 \text { \% площі України, є математична } \\
\text { симуляція. В основі останньої лежить морфометричний аналіз цифрових моделей } \\
\text { рельєфу, на базі якого виділяють ряд предикторів, які в подальшому аналізують на } \\
\text { предмет наявності зв'язків з існуючими картографічними ґрунтовими матеріалами } \\
\text { шляхом створення математичної предикативної моделі з використанням опорних точок } \\
\text { ландшафтів та приурочених до них ґрунтових таксонів. Виявлена різниця в якості } \\
\text { предикативних матеріалів з використанням індексу карра Коена (Соһеп's карра } \\
\text { соеffісіепt) дозволяє рекомендувати окремі джерела ЦМР як базові для такого роду } \\
\text { завдань. Проведено демонстрацію замкнутого виробничого циклу створення } \\
\text { предикативних ґрунтових картографічнихматеріалів на базі безкоштовного програмного } \\
\text { забезпечення - геоінформаційних систем GRASS та Qиапtum, мови статистичних } \\
\text { розрахунків R-Statistic та умовно-безкоштовного-векторизатора Еаsy Trace. }\end{array}$ \\
\hline
\end{tabular}

*E-mail: v.cherlinka@chnu.edu.ua

Форма цитування: Черлінка В.Р., Дмитрук Ю.М., Бодян Ю.Г. Вплив джерел ЦМР на якісні показники предикативних карт грунтового покриву. Агрохімія і грунтознавство. Міжвід. тем. наук. збірник. Вип. 90. Харків: ННЦ “ІҐА ім. О.Н. Соколовського”. 2020. C. 36-46. (Англ.). DOI: https://doi.org/10.31073/acss90-04.

УДК 631.4:551.4:004.942

\section{Влияние источников цифровой модели рельефа на качественные показатели предикативных карт почвенного покрова}

\author{
В. Р. Черлинка ${ }^{1 *}$, Ю. М. Дмитрук ${ }^{1}$, Ю. Г. Бодян ${ }^{2}$
}

1Черновицкий национальный университет им. Ю. Федьковича, Черновцы, Украина

2Черновицкий филиал ГП «Институт охраны почв Украины», Черновцы, Украина

Целью исследования было выявить влияние цифровых моделей рельефа (ЦМР) разного происхождения на качественные характеристики прогнозных карт почвенного покрова или картограмм агропроизводственных групп почв при использовании технологии предикативного моделирования. Проанализирована текущая ситуация с крупномасштабными почвенными картографическими данными в Украине и показано, что самым быстрым и экономически эффективным способом заполнения пробелов для обеспечения сплошного картографического покрытия необследованных территорий, площадь которых составляет 33\% площади территории Украины, является математическая симуляция. В основе последней лежит морфометрический анализ цифровых моделей рельеса, на базе которого выделяется ряд предикторов, которые в дальнейшем анализируются на предмет наличия связей с существующими картографическими почвенными материалами путем создания математической предикативной модели с использованием опорных точек ландшафтов и приуроченных к ним почвенных таксонов. Выявленные различия в качестве предикативных материалов с использованием индекса kappa Коена (Cohen's kappa coefficient) дают основание рекомендовать отдельные источники ЦМР как базовые для такого рода работ. Продемонстрирован замкнутый производственный цикл создания предикативных почвенных почвенных картографических материалов на базе бесплатного программного обеспечения геоинформационных систем GRASS и Quantum, языка статистических расчетов R-Statistic и условно- 
бесплатного - векторизатора Easy Trace.

Ключевые слова: карта почв; картограмма агропроизводственных групп почв; моделирование; морфометрические параметры; предикативные алгоритмы; цифровая модель рельефа; ЦМР. 